\title{
BMJ Open Use of the Diabetes Medication Choice Decision Aid in patients with type 2 diabetes in Greece: a cluster randomised trial
}

Thomas Karagiannis, ${ }^{1,2}$ Aris Liakos, ${ }^{1,2}$ Megan E Branda, ${ }^{3}$ Eleni Athanasiadou, ${ }^{1}$ Maria Mainou, ${ }^{1,2}$ Panagiota Boura, ${ }^{2}$ Dimitrios G Goulis, ${ }^{4}$ Annie LeBlanc, ${ }^{5}$ Victor M Montori, ${ }^{6}$ Apostolos Tsapas ${ }^{1,2,7}$

To cite: Karagiannis T, Liakos A, Branda ME, et al. Use of the Diabetes Medication Choice Decision Aid in patients with type 2 diabetes in Greece: a cluster randomised trial. BMJ Open 2016;6:e012185. doi:10.1136/bmjopen-2016012185

- Prepublication history and additional material is available. To view please visit the journal (http://dx.doi.org/ 10.1136/bmjopen-2016012185).

Received 6 April 2016 Revised 15 July 2016 Accepted 29 September 2016

CrossMark

For numbered affiliations see end of article.

Correspondence to Dr Thomas Karagiannis; tkaragian@auth.gr

\section{ABSTRACT}

Objective: To assess the efficacy of the Diabetes Medication Choice Decision Aid among patients with type 2 diabetes in Greece.

Design: Open-label cluster randomised controlled trial. Setting: Primary and secondary care practices across Greece.

Participants: 5 sites allocated to the decision aid ( $n=101$ patients) and 4 sites to control ( $n=103$ patients).

Intervention: Clinicians and patients in the intervention arm used a decision aid, based on outcomes that both consider important when choosing among antihyperglycaemic medications. Patients in the control arm received usual care.

Outcome measures: The primary outcome was patient's level of decisional comfort after the initial clinical encounter. Secondary outcomes included patient's knowledge about type 2 diabetes and medications, and patient's and clinician's satisfaction. Adherence to prescribed antihyperglycaemic medication and change in glycated haemoglobin were assessed at 24 weeks.

Results: Patients in both arms had similar scores in overall decisional comfort (mean difference between the usual care and decision aid arms $-6.9,95 \% \mathrm{Cl}-21.5$ to 7.7) and its subscales. Patients' knowledge was high in both arms (mean difference $2.3 \%, 95 \% \mathrm{Cl}-15.7 \%$ to $20.4 \%$ ). Patients and clinicians in both groups were equally satisfied with the decision-making. No significant difference in medication adherence and glycaemic control was found across arms. Clinicians found the decision aid useful and reported that its integration in their daily routine was easy.

Conclusions: The decision aid was implemented and positively received in the clinical setting in Greece, in line with the patient-centred approach endorsed by current guidelines. However, this trial yielded imprecise results in terms of patient outcomes. Further research is needed to investigate the interaction between the patient and the clinician in order to clarify the association between the use of decision aids and implementation of shared decision-making.

Trial registration number: NCT01861756. Pre-results.

\section{Strengths and limitations of this study}

- This is a practical cluster randomised trial promoting a patient-centred approach in patients with type 2 diabetes in Greece.

- We implemented a decision aid, originally developed and successfully tested in the USA, in the care of patients with type 2 diabetes in Greece.

- We did not directly evaluate the fidelity of use of the decision aid and the extent to which shared decision-making took place during the consultation.

\section{INTRODUCTION}

Most patients with type 2 diabetes mellitus (T2DM) will eventually require several antihyperglycaemic drugs or insulin to maintain glycaemic control. ${ }^{1}$ Available agents differ in the ways they may affect the risk for hypoglycaemia, body weight, the frequency and route of administration, or treatment cost. ${ }^{1-3}$ Moreover, it is questionable whether intensified treatment compared with routine care can actually reduce mortality or cardiovascular events in patients with T2DM. ${ }^{4}$ What is best for each patient will depend on each person's circumstances and what matters most to each one. Patients, however, rarely participate in selecting their antidiabetic medications, do not know their relative benefits and harms, and this may lead to ineffective decisions and poor medication adherence. ${ }^{56}$

Engaging patients in the decision-making process represents a path forward, justified in part on the premise that patients will experience the harms and costs of these choices. This patient-centred approach is endorsed by the American Diabetes Association (ADA) and the European Association for the Study of Diabetes (EASD) in their latest position statements. ${ }^{1} 7$ 
Moreover, the National Institute for Health and Care Excellence (NICE) in the UK has recently issued guidance highlighting the importance of involving patients with chronic conditions, including T2DM, in making decisions about medicines. ${ }^{8}$ How to achieve this vision, however, is not specified in these policy documents.

Decision aids are tools that can facilitate a patientcentred approach when presenting research evidence to patients, and have been used in many countries, including the UK, the USA and Canada. ${ }^{9-11}$ They have been shown to improve patients' knowledge and reduce decisional conflict when choosing among different treatment options for various healthcare conditions. ${ }^{9}$ Ideally, when using a decision aid during counselling, both the patient and the clinician share information, values and preferences and can potentially reach a decision together, through a shared decision-making process. ${ }^{12}$

The Diabetes Medication Choice Decision Aid (available at https://diabetesdecisionaid.mayoclinic.org) consists of seven cards that present information on diabetes-related outcomes that patients and clinicians consider important when choosing among different antihyperglycaemic medications. ${ }^{13}{ }^{14}$ Originally developed and evaluated by the Knowledge and Evaluation Research Unit in Mayo Clinic, the decision aid has also been successfully tested in cluster randomised trials in the USA. ${ }^{13-16}$ In this study, we aimed to implement and assess the efficacy of the Diabetes Medication Choice Decision Aid among patients with T2DM in Greece.

\section{METHODS}

Study design

We conducted an open-label cluster randomised controlled trial aiming to implement and evaluate the efficacy of the Diabetes Medication Choice Decision Aid in patients with T2DM in primary and secondary care practices in Greece. A cluster randomisation design was chosen to prevent 'contamination' by preference of patient or clinician. ${ }^{17}$ The level of randomisation was at the practice. The trial consisted of an initial patient-clinician encounter and two follow-up visits at 12 and 24 weeks. Owing to the nature of the intervention, clinicians and patients were not blinded. However, by virtue of how we designed the informed consent procedures, patients were kept masked to the study goals and main hypotheses. The recruitment period of patients within each participating practice was originally planned to last a total of 6 months, but was extended by an additional 3 months, as the patient recruitment rate was lower than expected. The study protocol and procedures were approved by the Bioethics Committee of the Medical School of the Aristotle University of Thessaloniki. The trial is registered at clinicaltrials.gov (NCT01861756).

\section{Recruitment of practices}

Eligible practices were selected based on the ability to participate in the study (ie, if they had at least one physician and an additional healthcare professional interested in participating), as well as having an on-site coordinator until completion of the trial. Eligible healthcare professionals from participating practices were physicians, nurse practitioners or physician assistants who provided care for patients with T2DM. We recruited practices with different geographical distribution across Greece. Eight practices agreed to participate in the study and were initially randomised. Of these, six were located in urban areas, while two were rural practices. Four practices provided primary care and the other four provided secondary care. However, shortly after trial initiation, one site randomised to the decision aid arm was shut down and was unable to recruit patients due to austerity measures in Greece. Thus, an additional practice with similar characteristics (secondary care located in an urban area) was enrolled and allocated to the decision aid arm. As a result, our analyses include data from five practices allocated to the decision aid and four practices allocated to usual care. Characteristics of all participating practices are presented in online supplementary table S1.

\section{Patient population}

With the intention of maximising the applicability of our results, we adopted a pragmatic approach by applying broad and inclusive patient eligibility criteria. We enrolled adults who have had T2DM for more than 1 year, agreed to be followed for at least 24 weeks by the participating clinician and were able to speak Greek at a level necessary to complete the patient surveys and ensure involvement in the decision-making process. Additionally, eligible patients had glycated haemoglobin (HbAlc) levels between $7.5 \%$ and $10 \%$, required treatment intensification and had more than one available treatment option, as judged by their clinician. Consecutive patients who visited each participating site were assessed for eligibility by the site's physician during their daily practice. Patients fulfilling the eligibility criteria were then invited by the physician to participate in the study. Those who agreed to participate and provided written informed consent were then recruited by the physician and enrolled in the study.

In addition to the demographic and clinical information necessary to enrol a patient (ie, age, sex, duration of T2DM), we also determined patients' socioeconomic status by collecting information about their education level and their current working and marital status. Moreover, baseline health-related quality of life was assessed with the use of the EQ-5D visual analogue scale, which has been previously used in diabetes trials and has also been validated in Greek patients. ${ }^{18}$

\section{Sample size}

Sample size calculation was based on results from the Statin Choice study, a cluster randomised trial that evaluated decisional quality comparing a decision aid to usual care. ${ }^{19}$ This study reported a 9.8 point difference in 
decisional quality with an SD of 16.9 and 14.1 for the usual care and decision aid groups, respectively. ${ }^{19}$ We planned to recruit 30 patients per practice, with a total recruitment target of 120 patients per study arm, in order to ensure $80 \%$ power to detect a difference of 9.8 points or greater in decision quality between the two groups, at a significance level of 0.05 with a two-sided t-test, using the following assumptions: (1) variances are as reported in the Statin Choice study; ${ }^{19}$ (2) there is a modest correlation of outcomes across clinicians and practices represented by an intracluster correlation coefficient (ICC) of $0.05 ;^{20}$ (3) there is a variance inflation or design effect factor $[1+(\mathrm{n}-1) \times \mathrm{ICC}]$, where $\mathrm{n}$ is the number of patients per cluster; and (4) an attrition rate of $20 \%$.

\section{Randomisation procedure}

Eligible practices were matched based on level of care (primary or secondary) by the study statistician, and were randomly allocated within each pair, using a computer-generated allocation sequence, to either the use of the Diabetes Medication Choice Decision Aid or to usual care. Since there was more than one pair per level, the statistician paired the sites without study team input. This seemed appropriate to avoid bias, as the statistician had no contact with participating sites and no information on them beyond information for randomisation.

\section{Intervention}

At practices allocated to the intervention arm, clinicians and patients used the Greek version of the Diabetes Medication Choice Decision Aid during the initial clinical encounter. It consists of seven cards that simply display the benefits and harms of commonly used antidiabetic medication classes across domains that patients and clinicians often consider important when choosing among several treatment options (reduction in HbAlc, weight change, hypoglycaemia, main adverse effects, treatment cost, daily sugar testing and drug-related daily routine) ${ }^{13}{ }^{14}$ During the consultation, the patient is presented with the seven cards by the physician and is asked which of the cards they would prefer to discuss. Ideally, after reviewing the chosen cards and discussing about alternative medication options, both the patient and the physician arrive at the medication choice that best matches the patient's preferences, at the end or right after the consultation. ${ }^{14}$

We translated the decision aid into Greek and updated the cost card to reflect the availability of generics, price and reimbursement procedures in Greece. Two different investigators translated all seven cards independently, while differences were resolved by consensus and arbitrated by a third investigator. Use of the decision aid was demonstrated to the physicians in practices allocated to the intervention arm, by a study team member through a short ( $\sim 1$ hour) in-person discussion and a brief video clip developed by Mayo Clinic and subtitled in Greek. Allied health professionals (nurses and physician assistants) were only trained in study procedures (ie, delivery of questionnaires, etc).

\section{Comparison}

Patients in practices assigned to the control arm received usual care for T2DM in Greece, without the use of the decision aid. Patients with T2DM in Greece are treated both in primary and secondary care, and have universal access and reimbursement for all therapeutic options described in the ADA/EASD position statement ${ }^{7}$ and approved by the European Medicines Agency. In fact, the latest Greek National Diabetes Guidelines, issued by the Hellenic Diabetes Association in 2013, are reflective of diabetes care in Greece and do not differ from the ADA/EASD position statement. ${ }^{7}$

\section{Outcome measures}

\section{Quality of the decision-making process}

Our primary outcome was patients' decisional comfort assessed immediately after the first clinical encounter using a 13-item modified Decisional Conflict Scale, which included 10 items that covered three (informed, support and effective subscales) of the five subscales from the original 16-item Decisional Conflict Scale, a validated instrument commonly used in trials evaluating the impact of decision supporting interventions. ${ }^{21}$ We excluded the six items covering the uncertainty and values clarity subscales from the original Decisional Conflict Scale and included three additional items instead. The 13 items chosen were thought to be most applicable to the study goals and are presented in online supplementary table S2. Of note, when reporting our results, the scale was inverted, so that higher scores were indicators of more comfort, instead of conflict.

\section{Patients' knowledge and satisfaction}

We assessed patients' knowledge using a six-item questionnaire addressing general knowledge about T2DM management and medications, completed by the patient after the baseline visit (see online supplementary table S3) ${ }^{16}$ In addition, we assessed patients' satisfaction with decision-making, as measured with 2 items from the 13-item modified Decisional Conflict Scale, asking patients to assess the extent to which they felt satisfied with their decision and the conversation they had with their clinician (see online supplementary table S4).

\section{Adherence to antidiabetic medication and clinical outcomes}

Information about adherence to prescribed antidiabetic drugs was assessed through patient self-reporting and by accessing electronic pharmacy records starting 8 weeks before the baseline visit and spanning to 24 weeks after it. For self-reported adherence, we used the Haynes single question that inquires whether the patient has missed at least one dose in the past week. ${ }^{22}$ We calculated medication adherence from electronic pharmacy records using the percentage of days covered, defined as 
the number of days a patient had a supply of each medication divided by the number of days of eligibility of that medication. ${ }^{23}$ In addition, patients' HbAlc was recorded at baseline and at 12 and 24 weeks, while body mass index was measured at baseline and at 24 weeks.

\section{Clinician outcomes}

The clinician's satisfaction with decision-making was measured by a simple question answered after the index encounter (see online supplementary table S4). Additionally, in the intervention arm, clinicians completed three additional questions regarding the ease of using the decision aid and incorporating it into their daily practice.

\section{Statistical analysis}

We conducted our analysis following the intentionto-treat principle, with the exception of medical adherence and clinical outcomes which were analysed based on completed data available. Analysis conducted accounted for the clustering (practice) in the study design. Missing self-reported patient outcomes were imputed to the overall mean for continuous outcomes and to 'neither agree/disagree' for satisfaction. Patient characteristics were compared between the intervention and control groups using the cluster-adjusted t-test and $\chi^{2}$ test. This method appropriately accounts for the clustering effect of patients within practices. ${ }^{24}$ No imbalances were found, so all analyses conducted take into account the clustering, but is unadjusted otherwise. Moreover, in order to explore the effect, on the primary outcome (overall decisional comfort and its three subscales), of the addition of a practice after the initial randomisation of eight practices, we conducted a sensitivity analysis excluding data for patients recruited by the ninth practice. We report adjusted means with 95\% CIs, when applicable. Study data were recorded using the Research Electronic Data Capture (REDCap) system. ${ }^{25}$ Statistical analysis was conducted using Stata (StataCorp. 2015. Stata Statistical Software: Release V.14. College Station, Texas: StataCorp LP) or SAS/STAT software.

\section{RESULTS}

\section{Participant flow and characteristics}

Participant flow is depicted in figure 1. Between May 2013 and February 2014, a total of 204 patients (101 in the decision aid arm and 103 in the usual care arm) were enrolled and are included in the analysis of the primary outcome. More patients were recruited in primary care practices (115 patients) compared with secondary care practices (89 patients; see online supplementary table S1). Patients' baseline characteristics were similar between the decision aid and the usual care arms (table 1).

\section{Patient outcomes}

After the initial patient-clinician encounter, patients in both arms were very comfortable with the decision (mean difference in decisional comfort between the usual care and decision aid arms $-6.9,95 \%$ CI -21.5 to 7.7). Similarly, there was no difference in the informed, support and effective decision subscales (table 2). Furthermore, in a sensitivity analysis, the exclusion of the practice added to the study after the initial randomisation had occurred did not have any impact on the findings regarding the primary outcome (see online supplementary table S5).

Patients' knowledge was also similar in both groups (mean difference $2.3 \%$, 95\% CI $-15.7 \%$ to $20.4 \%$ ). Moreover, patient satisfaction with the decision and the conversation with the clinician did not differ between the decision aid arm and usual care (table 2).

In both arms, only a small proportion of patients reported missing at least one medication dose in the past week (table 3). Percentage of days covered was assessed in 81 and 79 patients allocated to the decision aid and usual care arms, respectively, and was similarly high in both groups (mean difference $2.6 \%, 95 \%$ CI $-6.7 \%$ to $11.9 \%$; table 3 ). No significant difference in HbAlc was found across arms (table 3 ).

\section{Clinician outcomes}

Clinicians in both arms were equally satisfied with the decision-making process (table 4). Clinicians in the intervention arm reported using the decision aid in 85 $(87 \%)$ of the encounters. The main reasons for not using the decision aid were time constraints $(n=5)$ or belief that the patient was not interested $(n=2)$ or would not benefit $(n=3)$ from using the decision aid. Overall, most clinicians found that the use of the decision aid and its integration in practice was easy and reported that they would definitely or probably ( 41 and 38 , respectively, (94\%)) use a similar decision aid for their other patients.

\section{Deviations from the study protocol}

In the study protocol, we report decisional quality as our primary outcome, defined as decisional conflict measured with a modified 13-item version of the Decisional Conflict Scale. In our report, decisional conflict values were inverted to decisional comfort values, an approach also adopted in other studies evaluating decision aids. ${ }^{26}$

Moreover, in the study protocol, we report collecting data on patients' annual household income, family size and health literacy. However, before trial initiation, we removed these items for practical reasons, to reduce the burden for patients. Therefore, health literacy was not assessed, while socioeconomic status was assessed by collecting information solely about patients' educational level, current working and marital status.

Finally, we had asked study coordinators at each practice to keep a record of all patients invited to the study and of those who declined to participate. However, 


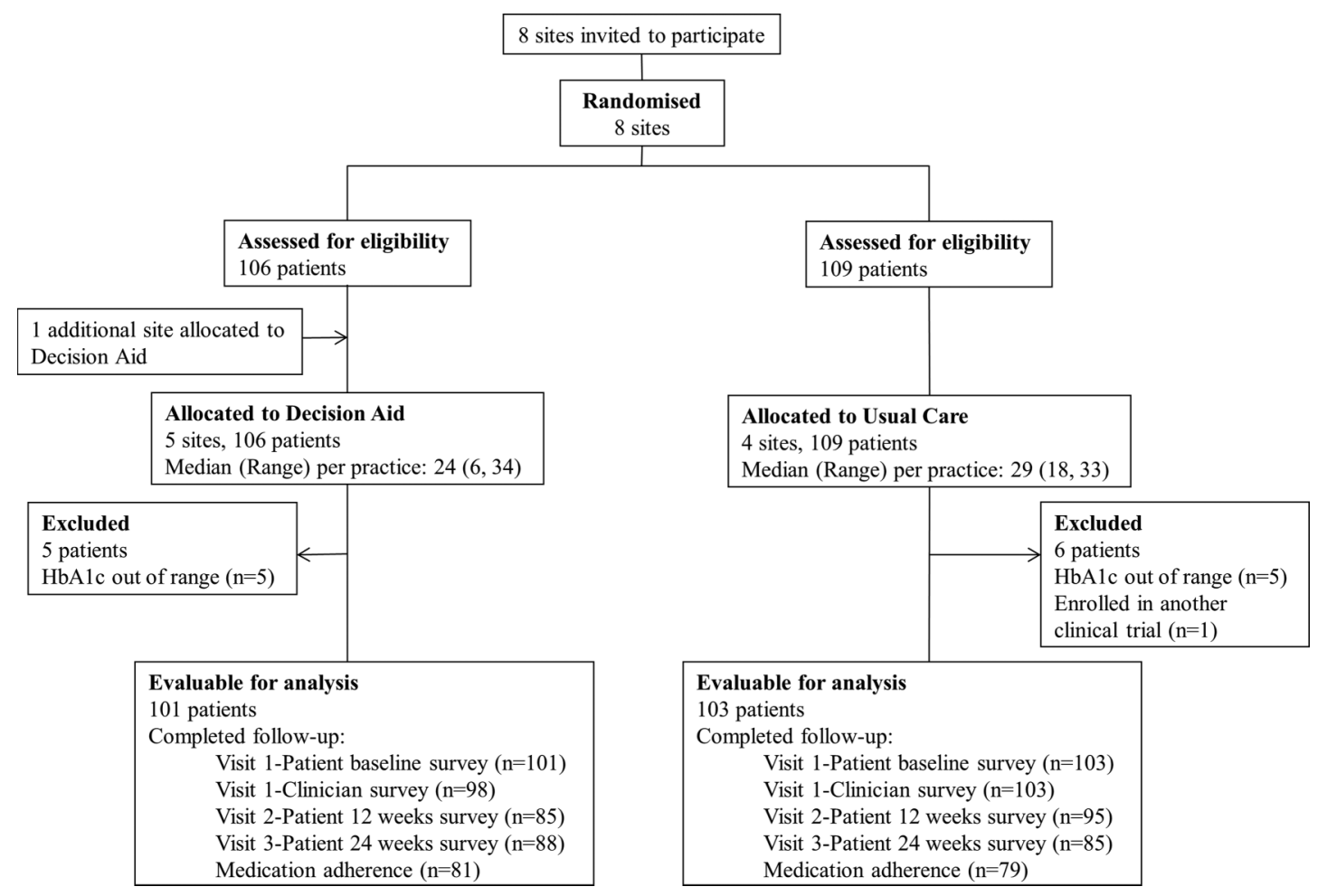

Figure 1 Participant flow diagram. HbA1c, glycated haemoglobin.

investigators involved did not adhere to the suggested practice (claiming it was impractical in their daily routine). Therefore, it is unknown how many patients were initially invited to each practice and how many of these declined participation.

\section{DISCUSSION}

In this cluster randomised trial, we implemented the Diabetes Medication Choice Decision Aid in primary and secondary care practices across Greece. While clinicians expressed a positive perception of their experience using the decision aid with their Greek patients and integrating it into their workflow, this trial yielded imprecise results in terms of patient outcomes. In particular, no significant differences were observed between the decision aid and usual care in decisional comfort, patients' knowledge, satisfaction with decision-making, glycaemic control or medication adherence.

\section{Strengths and limitations}

To the best of our knowledge, this is the first randomised trial to assess a decision aid in patients with T2DM in Greece. ${ }^{9}$ One could assume that in countries with limited experience in the implementation of decision aids, clinicians would probably be reluctant or less enthusiastic to adopt such an approach in their practice. However, our study demonstrated that the Diabetes Medication Choice Decision Aid was well received by clinicians who had no training in the use of decision aids prior to this trial. Furthermore, we used a cluster randomised design combined with a practical approach, by applying less stringent eligibility criteria and recruiting both primary and secondary care practices that provided care for patients with T2DM across Greece. This was implemented as the Greek healthcare system endured severe cuts as part of austerity measures to overcome a severe economic crisis. Additionally, we used a decision aid which is easily comprehensible to patients, consisting of simple graphic displays. Moreover, the decision aid has been designed in accordance with several of the International Patient Decision Aid Standards (IPDAS) criteria, and following input from patients' focus groups during the decision aid development process. ${ }^{1327}$

Nevertheless, our study has some important limitations. We did not evaluate the actual interaction between patients and clinicians, by means of recordings of the consultations and by applying a relevant validated instrument, such as the OPTION scale, to these recordings. ${ }^{28}$ Instead, we assessed the use of the decision aid with postintervention surveys; thus, it is impossible to assess the extent to which the decision aid was actually used in such a way that facilitates shared decisionmaking. ${ }^{29}$ Notably, many clinicians believe that they practise shared decision-making; however, research evidence suggests that there are misconceptions in healthcare professionals regarding the nature and goal of shared decision-making. ${ }^{30}{ }^{31}$ Moreover, use of the 
Table 1 Baseline characteristics of patients

\begin{tabular}{|c|c|c|c|}
\hline Characteristics & Usual care $(\mathrm{N}=103)$ & Decision aid $(\mathrm{N}=101)$ & ICC \\
\hline Age, mean (SD) & $64.4(11.3)$ & $65.8(11.0)$ & 0.116 \\
\hline Females, N (\%) & $61(59.2)$ & $55(54.5)$ & 0.000 \\
\hline \multicolumn{4}{|l|}{ Diabetes duration, N (\%) } \\
\hline$<5$ years & $22(21.4)$ & $27(26.7)$ & \multirow[t]{3}{*}{0.091} \\
\hline $5-10$ years & 27 (26.2) & $20(19.8)$ & \\
\hline$>10$ years & $53(51.5)$ & $53(52.5)$ & \\
\hline \multicolumn{4}{|l|}{ Education level, $\mathrm{N}(\%)$} \\
\hline$\leq$ High school degree & $85(82.5)$ & $62(61.3)$ & \multirow[t]{4}{*}{0.136} \\
\hline Technical school & $9(8.7)$ & $12(11.9)$ & \\
\hline College & $5(4.85)$ & $22(21.8)$ & \\
\hline Other & $4(3.9)$ & $2(2.0)$ & \\
\hline \multicolumn{4}{|l|}{ Working status, N (\%) } \\
\hline Not working at present & $84(81.6)$ & 78 (77.2) & \multirow[t]{3}{*}{0.046} \\
\hline Working part-time for pay & $1(1.0)$ & $5(5.0)$ & \\
\hline Working full-time for pay & $17(16.5)$ & $16(15.8)$ & \\
\hline \multicolumn{4}{|l|}{ Marital status, $\mathrm{N}(\%)$} \\
\hline Married & 75 (72.8) & 74 (73.2) & \multirow[t]{5}{*}{0.002} \\
\hline Divorced & $2(1.9)$ & $3(3.0)$ & \\
\hline Not married & $3(2.9)$ & $4(4.0)$ & \\
\hline Widowed & $21(20.4)$ & $18(17.8)$ & \\
\hline Separated & $2(1.9)$ & $1(1.0)$ & \\
\hline \multicolumn{4}{|l|}{ Years seeing clinician } \\
\hline First visit & $25(24.3)$ & $48(47.5)$ & \multirow[t]{4}{*}{0.271} \\
\hline$<1$ year & $22(21.4)$ & $18(17.8)$ & \\
\hline $1-5$ years & $35(34.0)$ & $21(20.8)$ & \\
\hline$>5$ years & $21(20.4)$ & $11(10.9)$ & \\
\hline Current smoker, N (\%) & 27 (26.2) & $18(17.8)$ & 0.002 \\
\hline $\begin{array}{l}\text { EQ-5D visual analogue scale }(0-100,100=\text { best } \\
\text { quality of life), mean (SD) }\end{array}$ & $66.7(20.9)$ & $68.4(16.0)$ & 0.010 \\
\hline $\mathrm{HbA} 1 \mathrm{c}(\%)$, mean (SD) & $8.5(0.8)$ & $8.4(0.8)$ & 0.031 \\
\hline BMI $\left(\mathrm{kg} / \mathrm{m}^{2}\right)$, mean (SD) & $31.1(6.0)$ & $30.2(5.7)$ & 0.068 \\
\hline
\end{tabular}

decision aid might have been more effective if patients were given more time to process the information provided in order to reach more thoughtful conclusions about their preferences, after completion of the first clinical encounter. ${ }^{32} 33$

Another important limitation of this study is the addition of a ninth practice in the intervention arm in a non-random manner, after loss of one of the initially randomised practices. Nevertheless, removing this practice in a sensitivity analysis had no significant impact on the primary outcome results. In addition, outcome assessors were not blinded in our study, while certain limitations are related to the choice of our outcome measures. In particular, we assessed patients' overall decisional comfort using a modified 13-item version instead of the original validated 16-item Decisional Conflict Scale. ${ }^{21}$ Moreover, it is unknown whether the Decisional Conflict Scale can adequately capture the overall concept of shared decision-making. ${ }^{34}$ Furthermore, the decision aid used in our study includes information on short-term or surrogate outcomes, such as hypoglycaemic risk or effect on body weight, rather than hard clinical end points, such as mortality or cardiovascular outcomes.
Finally, we did not manage to recruit the expected number of 120 patients in each arm, while our results for the primary outcome yielded a value of ICC $=0.305$. Such a high value of ICC is indicative of low within-cluster variability, which limits the effective sample size in our study and further reduces the study's power to detect differences between intervention and control. ${ }^{35}$ As a result, both of these shortcomings significantly limit the inferences we can draw about the effect of the decision aid on the outcomes measured in this trial.

\section{Comparison with other studies}

The Diabetes Medication Choice Decision Aid has been previously assessed in two cluster randomised trials enrolling rural and suburban primary care practices in the USA. ${ }^{1416}$ Similarly to our study, clinicians using the decision aid in the USA found it easy to integrate it in their practice, while compared with usual care, the decision aid did not affect patient decisional comfort, satisfaction and medication adherence. As opposed to our study, use of the decision aid was associated with increased patient knowledge about T2DM and 
Table 2 Patient-reported quality of the decision-making process outcomes

\begin{tabular}{|c|c|c|c|c|c|}
\hline Outcomes* & Usual care $(\mathrm{N}=103)$ & Decision aid $(\mathrm{N}=101)$ & Mean difference & p Value & ICC \\
\hline $\begin{array}{l}\text { Overall decisional comfort; } \\
0-100,100=\text { no conflict } \\
(95 \% \mathrm{Cl})\end{array}$ & 75.1 (61.0 to 89.2 ) & 82.0 (70.0 to 94.0$)$ & $-6.9(-21.5$ to 7.7$)$ & 0.30 & 0.305 \\
\hline Informed subscale $(95 \% \mathrm{Cl})$ & $65.4(44.3$ to 86.5$)$ & 78.8 (60.9 to 96.8$)$ & $-13.4(-35.3$ to 8.4$)$ & 0.19 & 0.265 \\
\hline Support subscale $(95 \% \mathrm{Cl})$ & 77.6 (61.3 to 93.9$)$ & 80.7 (66.9 to 94.6$)$ & $-3.1(-20.0$ to 13.8$)$ & 0.67 & 0.301 \\
\hline Effective subscale $(95 \% \mathrm{Cl})$ & 80.5 (69.9 to 91.1$)$ & 85.2 (76.2 to 94.3$)$ & $-4.7(-15.7$ to 6.3$)$ & 0.35 & 0.197 \\
\hline $\begin{array}{l}\text { Knowledge; } 0-100,100=\text { all } \\
\text { correct answers }(95 \% \mathrm{Cl})\end{array}$ & $70.7 \%(53.4 \%$ to $88.1 \%)$ & $68.4 \%(53.6 \%$ to $83.2 \%)$ & $2.3 \%(-15.7 \%$ to $20.4 \%)$ & 0.77 & 0.234 \\
\hline \multicolumn{6}{|c|}{ Satisfaction with decision made, $\mathrm{N}(\%)$} \\
\hline Strongly agree & $37(35.9)$ & $52(51.5)$ & NA & 0.37 & 0.063 \\
\hline Agree & $58(56.3)$ & 38 (37.6) & & & \\
\hline $\begin{array}{l}\text { Neither agree nor } \\
\text { disagree }\end{array}$ & $7(6.8)$ & $11(10.9)$ & & & \\
\hline Disagree & $1(1.0)$ & $0(0)$ & & & \\
\hline Strongly disagree & $0(0)$ & $0(0)$ & & & \\
\hline \multicolumn{6}{|c|}{ Satisfaction with the conversation with the clinician, N (\%) } \\
\hline Strongly agree & $58(56.3)$ & $66(65.3)$ & NA & 0.54 & 0.105 \\
\hline Agree & $44(42.7)$ & $31(30.7)$ & & & \\
\hline $\begin{array}{l}\text { Neither agree nor } \\
\text { disagree }\end{array}$ & $1(1.0)$ & $4(4.0)$ & & & \\
\hline Disagree & $0(0)$ & $0(0)$ & & & \\
\hline Strongly disagree & $0(0)$ & $0(0)$ & & & \\
\hline
\end{tabular}

Table 3 Medication adherence and clinical outcomes

\begin{tabular}{|c|c|c|c|c|c|}
\hline Outcomes & $\begin{array}{l}\text { Usual care } \\
(\mathrm{N}=103)\end{array}$ & $\begin{array}{l}\text { Decision aid } \\
(\mathrm{N}=101)\end{array}$ & $\begin{array}{l}\text { Mean difference } \\
(95 \% \mathrm{Cl})\end{array}$ & $\begin{array}{l}p \\
\text { Value }\end{array}$ & ICC \\
\hline $\begin{array}{l}\text { Proportion of days covered for all antidiabetic } \\
\text { medications (SD) }\end{array}$ & $93.2(25.2)$ & $90.5(24.6)$ & $2.6(-6.7$ to 11.9$)$ & 0.52 & 0.054 \\
\hline Number analysed & 79 & 81 & & & \\
\hline \multicolumn{6}{|c|}{ Missed medicine in prior week, assessed at 12 weeks, $N(\%)$} \\
\hline Responders & 81 & 70 & NA & 0.35 & 0.063 \\
\hline None & $69(85.2)$ & $67(95.7)$ & & & \\
\hline Once & $4(4.9)$ & $1(1.4)$ & & & \\
\hline More than twice & $8(9.9)$ & $2(2.9)$ & & & \\
\hline \multicolumn{6}{|c|}{ Missed medicine in prior week, assessed at 24 weeks, $\mathrm{N}(\%)$} \\
\hline Responders & 63 & 80 & NA & 0.61 & 0.122 \\
\hline None & $55(87.3)$ & 75 (93.8) & & & \\
\hline Once & $2(3.2)$ & $3(3.7)$ & & & \\
\hline More than twice & $6(9.5)$ & $2(2.5)$ & & & \\
\hline Change in $\mathrm{HbA} 1 \mathrm{c}$ at 12 weeks (\%), mean (SD) & $-1.0(0.97)$ & $-0.6(0.96)$ & $-0.33(-0.7$ to 0.002$)$ & 0.051 & 0.008 \\
\hline Number analysed & 96 & 91 & & & \\
\hline Change in $\mathrm{HbA} 1 \mathrm{c}$ at 24 weeks (\%), mean (SD) & $-1.1(1.5)$ & $-0.85(1.5)$ & $-0.20(-0.8$ to 0.3$)$ & 0.41 & 0.053 \\
\hline Number analysed & 85 & 92 & & & \\
\hline BMI at 24 weeks $\left(\mathrm{kg} / \mathrm{m}^{2}\right)$, mean (SD) Number & $31.5(8.8)$ & $29.4(8.6)$ & $2.1(-1.1$ to 5.3$)$ & 0.17 & 0.058 \\
\hline analysed & 83 & 84 & & & \\
\hline
\end{tabular}

antidiabetic medications; however, this result should be interpreted with caution due to limited precision of the study, attributed to low recruitment status. ${ }^{16}{ }^{36}$ A recently published study explored the effect of another decision aid for T2DM focusing on shared goal setting and decision-making regarding treatment of risk factors. ${ }^{37}$ As stated by the authors, use of the decision aid was not implemented as intended, since less than half of the 
Table 4 Clinician outcomes

\begin{tabular}{|c|c|c|}
\hline Outcomes & $\begin{array}{l}\text { Usual } \\
\text { care } \\
(\mathrm{N}=103)\end{array}$ & $\begin{array}{l}\text { Decision } \\
\text { aid } \\
(\mathrm{N}=101)\end{array}$ \\
\hline \multicolumn{3}{|c|}{$\begin{array}{l}\text { Discussion about medications occurred in the encounter, } \\
\mathrm{N}(\%)\end{array}$} \\
\hline Yes & $102(100)$ & $97(99.0)$ \\
\hline No & $0(0)$ & $1(1.0)$ \\
\hline \multicolumn{3}{|c|}{ Satisfaction with discussion, N (\%)† } \\
\hline Completely & $18(17.7)$ & $34(34.7)$ \\
\hline Very & $61(59.8)$ & $49(50.0)$ \\
\hline Somewhat & $18(17.7)$ & $9(9.2)$ \\
\hline Poorly & $5(4.9)$ & $5(5.1)$ \\
\hline Not at all & 0 & $1(1.0)$ \\
\hline \multicolumn{3}{|l|}{ Use of the decision aid, $\mathrm{N}(\%)$} \\
\hline Yes & NA & $85(86.7)$ \\
\hline No & & $13(13.3)$ \\
\hline \multicolumn{3}{|c|}{ Reason for not using the decision aid, $\mathrm{N}(\%)$} \\
\hline $\begin{array}{l}\text { Patient already knew about } \\
\text { medications }\end{array}$ & NA & $1(7.7)$ \\
\hline Patient not interested & & $2(15.4)$ \\
\hline Not enough time & & $5(38.5)$ \\
\hline Patient would not benefit & & $3(23.0)$ \\
\hline Reason not specified & & $2(15.4)$ \\
\hline \multicolumn{3}{|c|}{ Delivery of the decision aid, $\mathrm{N}(\%)$} \\
\hline Very easy & NA & $40(47.1)$ \\
\hline Easy & & $33(38.9)$ \\
\hline Neither easy nor difficult & & $5(5.9)$ \\
\hline Difficult & & 7 (8.2) \\
\hline Very difficult & & $0(0)$ \\
\hline \multicolumn{3}{|c|}{ Integration of the decision aid for coworkers, $\mathrm{N}(\%)$} \\
\hline Very easy & NA & $31(36.5)$ \\
\hline Easy & & $34(40.0)$ \\
\hline Neither easy nor difficult & & $15(17.7)$ \\
\hline Difficult & & $5(5.9)$ \\
\hline Very difficult & & $0(0.0)$ \\
\hline \multicolumn{3}{|c|}{$\begin{array}{l}\text { 'Would you use a similar decision aid for patients with } \\
\text { other chronic conditions?', N (\%) }\end{array}$} \\
\hline Definitely & NA & $41(48.8)$ \\
\hline Probably & & $38(45.2)$ \\
\hline Not sure & & $4(4.8)$ \\
\hline Probably not & & $1(1.2)$ \\
\hline
\end{tabular}

participants reported receiving the basic elements of the intervention. ${ }^{37}$ Notably, a randomised controlled trial on patients with T2DM in Germany demonstrated that patients who received an informed shared decisionmaking programme intervention achieved higher levels of risk comprehension for prevention of myocardial infarction, compared with the control group. ${ }^{38}$

\section{Implications for practice and research}

There is considerable policy support for shared decisionmaking for chronic conditions, including T2DM, from many associations, agencies and research groups. ${ }^{1} 8$ 39-41 Even though the Diabetes Medication Choice Decision
Aid did not significantly affect outcomes in our study, its implementation was feasible in primary and secondary care practices in Greece.

Further work is required, focused on the interaction between the patient and the clinician, in order to clarify the association between the use of decision aids and shared decision-making. ${ }^{42}$ This can be achieved by applying observational methods during the patient-clinician consultation, such as videorecording the clinical encounter, and by implementing qualitative interviews in future studies that can help identify probable barriers to the efficient use of decision aids. Larger studies with a longer follow-up are required to identify subgroup populations that could possibly benefit more from the use of decision aids, such as patients with poor glycaemic control who exhibit low adherence to treatment at baseline. Furthermore, the role of decision aids remains to be examined in various cultural groups, as well as in countries where patients are less informed about their condition, less educated or get less involved in caring for their disease. Finally, future development of decision aids for T2DM should consider other elements apart from medication choice, such as goal setting, non-pharmacological options and incorporating data regarding prevention of other common comorbidities, including arterial hypertension, dyslipidaemia, obesity or chronic kidney disease. In fact, a recent randomised controlled trial on patients with T2DM has demonstrated promising results of an informed shared decision-making programme, including a decision aid, with regard to patients' risk comprehension for myocardial infarction. ${ }^{38}$

\section{CONCLUSION}

In this study, the Diabetes Medication Choice Decision Aid was implemented in the primary and secondary clinical settings, and positively received by clinicians in Greece, in line with the patient-centred approach for T2DM endorsed by ADA and EASD. ${ }^{1} 7$ There was no effect of the decision aid on the primary outcome of decisional comfort and on secondary outcomes. However, patient-centred care is essential, irrespective of its potential effect on patient outcomes, based on ethical grounds related to patient autonomy, patient knowledge about treatment options and trust in the patient-clinician relationship. ${ }^{39} 43$ This trial informs the feasibility of conducting clinic-based practical trials of patient-centred interventions, even in the turmoil of the Greek economic crisis.

Part of this study has been presented at the 50th EASD Annual Meeting, 15-19 September 2014, Vienna, Austria.

\section{Author affiliations}

${ }^{1}$ Clinical Research and Evidence-Based Medicine Unit, Aristotle University Thessaloniki, Thessaloniki, Greece

${ }^{2}$ Second Medical Department, Aristotle University Thessaloniki, Thessaloniki, Greece 
${ }^{3}$ Knowledge and Evaluation Research Unit, Division of Biomedical Statistics and Informatics, Department of Health Sciences Research, Mayo Clinic, Rochester, Minnesota, USA

${ }^{4}$ Unit of Reproductive Endocrinology, First Department of Obstetrics and Gynaecology, Aristotle University Thessaloniki, Thessaloniki, Greece ${ }^{5}$ Knowledge and Evaluation Research Unit, Division of Health Care Policy and Research, Department of Health Sciences Research, Mayo Clinic, Rochester, Minnesota, USA

${ }^{6}$ Knowledge and Evaluation Research Unit, Division of Endocrinology, Department of Medicine, Mayo Clinic, Rochester, Minnesota, USA

${ }^{7}$ Harris Manchester College, University of Oxford, Oxford, UK

Acknowledgements The authors thank the EuroQol Group for permission to use the EQ-5D visual analogue scale and all individual study collaborators of the nine participating sites (S Alexiadis, M Benroumpi, T Chatziadamidou, E Giannakopoulos, S Irakleianou, K Kazakos, I Mygdalis, E Pagkalos, D Papazoglou and N Tentolouris).

Collaborators S Alexiadis, M Benroumpi, T Chatziadamidou, E Giannakopoulos, S Irakleianou, K Kazakos, I Mygdalis, E Pagkalos, D Papazoglou, N Tentolouris.

Contributors TK, ALe, VMM and AT led the conception of the study. TK, ALi, ALe, VMM and AT led the design of the study protocol and MEB, MM, PB and DGG participated. AT applied for research funding. TK, MM and AT participated in the translation of the Diabetes Medication Choice Decision Aid and study surveys into Greek. TK, ALi, EA, MM and AT prepared and distributed the study material to participating sites. ALi and EA coordinated the data collection and TK participated. MEB led the statistical analysis and TK participated. All authors participated in the interpretation of the trial data. TK, ALi and AT had full access to the study data, and take responsibility for the integrity of the data and the accuracy of the data analysis. TK, MEB and AT drafted the article and all authors contributed to a critical revision of the manuscript. All authors have approved the final version of the manuscript. TK and $A T$ are the study guarantors.

Funding This study was funded by a European Foundation for the Study of Diabetes (EFSD) research programme in patient education supported by an educational grant from AstraZeneca/BMS in 2012.

\section{Competing interests None declared.}

Ethics approval This study was approved by the Bioethics Committee of the Medical School of the Aristotle University of Thessaloniki (meeting no. 3/ 23-06/2012)

Provenance and peer review Not commissioned; externally peer reviewed.

Data sharing statement No additional data are available.

Open Access This is an Open Access article distributed in accordance with the Creative Commons Attribution Non Commercial (CC BY-NC 4.0) license, which permits others to distribute, remix, adapt, build upon this work noncommercially, and license their derivative works on different terms, provided the original work is properly cited and the use is non-commercial. See: http:// creativecommons.org/licenses/by-nc/4.0/

\section{REFERENCES}

1. Inzucchi SE, Bergenstal RM, Buse JB, et al. Management of hyperglycemia in type 2 diabetes, 2015: a patient-centered approach: update to a position statement of the American Diabetes Association and the European Association for the Study of Diabetes. Diabetes Care 2015;38:140-9.

2. Saulsberry WJ, Coleman $\mathrm{Cl}$, Mearns ES, et al. Comparative efficacy and safety of antidiabetic drug regimens added to stable and inadequate metformin and thiazolidinedione therapy in type 2 diabetes. Int J Clin Pract 2015;69:1221-35.

3. Mearns ES, Saulsberry WJ, White CM, et al. Efficacy and safety of antihyperglycaemic drug regimens added to metformin and sulphonylurea therapy in type 2 diabetes: a network meta-analysis. Diabet Med 2015;32:1530-40.

4. Griffin SJ, Borch-Johnsen K, Davies MJ, et al. Effect of early intensive multifactorial therapy on 5-year cardiovascular outcomes in individuals with type 2 diabetes detected by screening
(ADDITION-Europe): a cluster-randomised trial. Lancet 2011;378:156-67.

5. Sepucha KR, Fagerlin A, Couper MP, et al. How does feeling informed relate to being informed? The DECISIONS survey. Med Decis Making 2010;30(5 Suppl):77S-84S.

6. Voorham J, Haaijer-Ruskamp FM, Wolffenbuttel BH, et al. Medication adherence affects treatment modifications in patients with type 2 diabetes. Clin Ther 2011;33:121-34.

7. Inzucchi SE, Bergenstal RM, Buse JB, et al. Management of hyperglycemia in type 2 diabetes: a patient-centered approach position statement of the American Diabetes Association (ADA) and the European Association for the Study of Diabetes (EASD). Diabetes Care 2012;35:1364-79.

8. National Institute for Health and Care Excellence. Medicines optimisation. The safe and effective use of medicines to enable best possible outcomes. 2015. http://www.nice.org.uk/guidance/ng5/ evidence/full-guideline-6775454

9. Stacey D, Legare F, Col NF, et al. Decision aids for people facing health treatment or screening decisions. Cochrane Database Syst Rev 2014;1:CD001431.

10. Frosch DL, Moulton BW, Wexler RM, et al. Shared decision making in the United States: policy and implementation activity on multiple fronts. Z Evid Fortbild Qual Gesundhwes 2011;105:305-12.

11. Légaré F, Stacey D, Forest PG, et al. Moving SDM forward in Canada: milestones, public involvement, and barriers that remain. Z Evid Fortbild Qual Gesundhwes 2011;105:245-53.

12. Stiggelbout AM, Van der Weijden T, De Wit MP, et al. Shared decision making: really putting patients at the centre of healthcare. BMJ 2012;344:e256.

13. Breslin M, Mullan RJ, Montori VM. The design of a decision aid about diabetes medications for use during the consultation with patients with type 2 diabetes. Patient Educ Couns 2008;73:465-72.

14. Mullan RJ, Montori VM, Shah ND, et al. The diabetes mellitus medication choice decision aid: a randomized trial. Arch Intern Med 2009;169:1560-8.

15. LeBlanc A, Ruud KL, Branda ME, et al. The impact of decision aids to enhance shared decision making for diabetes (the DAD study): protocol of a cluster randomized trial. BMC Health Serv Res 2012;12:130.

16. Branda ME, LeBlanc A, Shah ND, et al. Shared decision making for patients with type 2 diabetes: a randomized trial in primary care. BMC Health Serv Res 2013;13:301.

17. Fayers PM, Jordhoy MS, Kaasa S. Cluster-randomized trials. Palliat Med 2002;16:69-70.

18. Kontodimopoulos N, Pappa E, Niakas D, et al. Validity of the EuroQoL (EQ-5D) instrument in a Greek general population. Value Health 2008;11:1162-9.

19. Weymiller AJ, Montori VM, Jones LA, et al. Helping patients with type 2 diabetes mellitus make treatment decisions: statin choice randomized trial. Arch Intern Med 2007;167:1076-82.

20. Littenberg B, MacLean CD. Intra-cluster correlation coefficients in adults with diabetes in primary care practices: the Vermont Diabetes Information System field survey. BMC Med Res Methodol 2006;6:20.

21. O'Connor AM. Validation of a decisional conflict scale. Med Decis Making 1995;15:25-30.

22. Haynes RB, McDonald HP, Garg AX. Helping patients follow prescribed treatment: clinical applications. JAMA 2002;288:2880-3.

23. Hess LM, Raebel MA, Conner DA, et al. Measurement of adherence in pharmacy administrative databases: a proposal for standard definitions and preferred measures. Ann Pharmacother 2006;40:1280-8.

24. Donner A, Klar N. Design and analysis of cluster randomization trials in health research. London: Arnold, 2000.

25. Harris PA, Taylor R, Thielke R, et al. Research electronic data capture (REDCap) - a metadata-driven methodology and workflow process for providing translational research informatics support. $J$ Biomed Inform 2009;42:377-81.

26. LeBlanc A, Herrin J, Williams MD, et al. Shared decision making for antidepressants in primary care: a cluster randomized trial. JAMA Intern Med 2015:175:1761-70.

27. Volk RJ, Llewellyn-Thomas H, Stacey D, et al. Ten years of the International Patient Decision Aid Standards Collaboration: evolution of the core dimensions for assessing the quality of patient decision aids. BMC Med Inform Decis Mak 2013;13(Suppl 2):S1.

28. Melbourne E, Sinclair K, Durand MA, et al. Developing a dyadic OPTION scale to measure perceptions of shared decision making. Patient Educ Couns 2010;78:177-83.

29. Hargraves I, Montori VM. Decision aids, empowerment, and shared decision making. BMJ 2014;349:g5811.

30. Agoritsas $T$, Heen AF, Brandt L, et al. Decision aids that really promote shared decision making: the pace quickens. BMJ 2015;350: g7624. 
31. Hoffmann TC, Légaré F, Simmons MB, et al. Shared decision making: what do clinicians need to know and why should they bother? Med J Aust 2014;201:35-9.

32. Elwyn G, Miron-Shatz T. Deliberation before determination: the definition and evaluation of good decision making. Health Expect 2010;13:139-47.

33. Elwyn G, Frosch D, Thomson R, et al. Shared decision making: a model for clinical practice. J Gen Intern Med 2012;27:1361-7.

34. Gionfriddo MR, Leppin AL, Brito JP, et al. Shared decision-making and comparative effectiveness research for patients with chronic conditions: an urgent synergy for better health. J Comp Eff Res 2013;2:595-603.

35. Killip S, Mahfoud Z, Pearce K. What is an intracluster correlation coefficient? Crucial concepts for primary care researchers. Ann Fam Med 2004;2:204-8.

36. Ruud KL, Leblanc A, Mullan RJ, et al. Lessons learned from the conduct of a multisite cluster randomized practical trial of decision aids in rural and suburban primary care practices. Trials 2013;14:267.

37. Denig P, Schuling J, Haaijer-Ruskamp F, et al. Effects of a patient oriented decision aid for prioritising treatment goals in diabetes: pragmatic randomised controlled trial. BMJ 2014;349: g5651.

38. Buhse S, Mühlhauser I, Heller T, et al. Informed shared decision-making programme on the prevention of myocardial infarction in type 2 diabetes: a randomised controlled trial. $B M$ Open 2015;5:e009116.

39. Sheridan SL, Harris RP, Woolf SH. Shared decision making about screening and chemoprevention. A suggested approach from the U.S. Preventive Services Task Force. Am J Prev Med 2004;26:56-66.

40. Consumer Assessment of Healthcare Providers and Systems. Shared Decision-Making, 2016. https://cahps.ahrq.gov/ quality-improvement/improvement-guide/6-strategies-for-improving/ communication/strategy6i-shared-decisionmaking.html

41. Patient-Centered Outcomes Research Institute. 2016. http://www. pcori.org/

42. Tamhane S, Rodriguez-Gutierrez R, Hargraves I, et al. Shared decision-making in diabetes care. Curr Diab Rep 2015;15:112.

43. Shay LA, Lafata JE. Where is the evidence? A systematic review of shared decision making and patient outcomes. Med Decis Making 2015;35:114-31. 